\title{
Microwave-Assisted Synthesis and Characterization of Random Copolyesters
}

\author{
KAMALANATHAN TAMILARASAN ${ }^{1}$, R. NANTHINI ${ }^{1}$ N. PRAKASH ${ }^{2}$ \\ and N. G. RENGANATHAN ${ }^{2}$
}

${ }^{1}$ Department of Chemistry, Pachaiyappa's College, University of Madras, Chennai-30, India

${ }^{2}$ Department of Chemistry, Vel Tech Dr. RR \& Dr.SR Technical University, Chennai-62, India

knprakash08@gmail.com

Received 27 May 2013 / Accepted 10 July 2013

\begin{abstract}
Synthesis and characterization of polyesters containing chalcone chromosphers were carried out and their properties were studied. Chalcones are 1,3-diphenyl-2-propene-1-one in which two aromatic rings linked by a three carbon $\alpha, \beta$ - unsaturated carbonyl system. Synthesis has been carried out using microwave. The MW-assisted synthesis of co-polyesters has been effected using from propane1,3-diol and 1,4-cyclohexane dimethanol with various carboxylic acids in the presence of titanium isopropoxide as the catalyst. Microwave structural analysis and thermal properties of biodegradable diblock copolyesters were also performed. The synthesized polymers were characterized by solubility measurement and intrinsic viscosity methods. Results are presented and discussed.
\end{abstract}

Keywords: Poly(propane1,3-diol fumaric acid1,4-cyclohexanedicarboxylic acid), Poly(1,4cyclohexanedimethanol fumaric acid 1,4-cyclohexanedicarboxylic acid), Poly(propane1,3-diol fumaric acid 1,4-cyclohexanedimethanol), Poly condensation

\section{Introduction}

The family of polyesters comprises all polymers with ester functional groups in the polymer back bone ${ }^{1}$. In principle, the synthesis of polyesters or esters in the presence of large amount of water has only been studied by a few research groups. Saam et al. ${ }^{2-4}$ studied the poly condensation in suspension of hydrophobic diol and diacid compounds using different sulfonate surfactants. They defined the principles of such polycondensation and showed that polyesters of low molecular weight could be obtained in aqueous media. More recently, Manabe et $a l^{5-7}$ studied the esterification of hydrophobic mono-functional compounds in aqueous suspension. Copolyesters, obtained from a multiplicity of reactions having the component groups linked in a random or statistical order, are termed random co polyesters. They retain their strength, clarity and other mechanical properties, despite being exposed to a variety of chemicals that typically affect other materials, such as polycarbonates. 
This includes their versatility and flexibility which enhances their application effectively in the design of high-volume, low cast parts as well as critical, more expensive component parts. Phase transfer catalysis is a synthetic technique which involves transport of an organic or inorganic salt form a solid or aqueous phase into an organic/liquid phase where reaction with an organic soluble substrate takes place. Chalcones are 1,3-diphenyl-2-propene-1-one, in which two aromatic rings are linked by a three carbon $\alpha, \beta$-unsaturated carbonyl system. They possess conjugated double bonds and a completely delocalised $\pi$-electron system on both benzene rings ${ }^{8}$. Molecules possessing such system have relatively low redox potentials and have a greater probability of undergoing electron transfer reactions. Most of the research studies involved only the polymers with side chain chalcone units. There are only few reports on soluble polymers with chalcone units in the main chain. Incorporation of flexible aliphatic chain segments into the polymer backbone improves the solubility. The present work deals with the synthesis and characterization of polyesters containing chalcone chromospheres in the main chain and study of their properties.

\section{Experimental}

Melting points were determined with open capillary tube on a Gallenkamp (variable heater) melting point apparatus and were uncorrected. Infra red spectra were recorded as $\mathrm{KBr}$ pellets on a buck spectrometer. ${ }^{1} \mathrm{H}$ and ${ }^{13} \mathrm{C}$ NMR were run on a Bruker-AC-250 and JEOL-JNMGX 300-MHz spectrometer (in ppm relative to $\mathrm{Me} 4 \mathrm{Si}$ and $\mathrm{H}_{3} \mathrm{PO}_{4}$ ). The elemental analysis $(\mathrm{C}, \mathrm{H}, \mathrm{N})$ of compounds were performed using a carbon-hydrogen elemental analyzer. The microwave-assisted syntheses were carried out in domestic oven, Panasonic $400 \mathrm{~W}$. Solvents used were of reagent grade and when necessary, were purified and dried by standard methods.

\section{Microwave method}

The reaction mixture was taken in round-bottom flask placed in a microwave oven and irradiated at $400 \mathrm{~W}$ for $20 \mathrm{~min}$ with addition of catalyst TTiPO. The solid product was dried and dissolved in chloroform then filtered and re-precipitate from methanol.

\section{Synthesis of polyesters}

The MW-assisted synthesis of co-polyesters from propane 1, 3-diol and 1, 4-cyclohexane dimethanol with various carboxylic acids in the presence of titanium isopropoxide as the catalyst. The influence of reaction time, reaction temperature, catalyst concentration and monomer ratio on the polymer yield and the molecular weight studied for bulk and solution poly condensation. A reaction temperature of $29{ }^{\circ} \mathrm{C}$ two dicarboxylic acid/diol ratio of 1:1:2 and a MW irradiation time of $20 \mathrm{~min}$ on $300-500 \mathrm{~W}$. A comparison with conventional heating under these conditions. Showed 10 fold increases of reaction rate under MW conditions.

\section{Results and Discussion}

\section{Molecular weight}

In order to improve the thermal and mechanical properties of the poly (estercarbonate)s, chain-extension products (PCDMFCDC, PPFCDM and PPFCDC) were also synthesized starting from dihydroxy-terminated oligomers obtained by the etherification of succinic acid in the presence of 1,3-propanediol and 1,4-cyclohexanedimethanol (PCDMFCDC, PPFCDM and PPFCDC $)^{9}$. Molecular weights and molecular weight distributions of the polymers obtained, as well as the transition temperatures are reported in Table 1. 
Table 1. Molecular and thermal characterization of poly (ester-carbonates)

\begin{tabular}{cccccccc}
\hline S. No & Sample & $\overline{\mathrm{Mn}_{\mathrm{n}}}$ & $\overline{\mathrm{Ww}}$ & $\mathrm{PD}$ & $\mathrm{T}_{\mathrm{g}}{ }^{0} \mathrm{C}$ & $\mathrm{T}_{\mathrm{m}}{ }^{0} \mathrm{C}$ & $D \mathrm{DH} / \mathrm{g}$ \\
\hline 1. & PCDMFCDC & 5900 & 10300 & 1.74 & 67.47 & 95.27 & 27.81 \\
2. & PPFCDM & 4900 & 9100 & 1.84 & 62.30 & 96.67 & 14.46 \\
3. & PPFCDC & 5300 & 8900 & 1.67 & 61.77 & 97.29 & 13.55 \\
\hline
\end{tabular}

Molecular weights of the long chain branched low $\mathrm{T}_{\mathrm{g}}$ polyesters were characterized using Size Exclusion Chromatography (Table 1). Characterization of the molecular weight and branching index indicated that synthesis of long chain branched low $\mathrm{T}_{\mathrm{g}}$ polyesters in the presence of mono functional reagents retarded gel formation and permitted synthesis of gel free polymers having higher degrees of branching. Moreover, the presence of end cappers allowed incorporation of TMT up to $3.2 \mathrm{~mol} 1 \%$. As shown in Table 1, reaction of higher levels of tri functional reagent resulted in synthesis of polymers with higher degrees of branching as depicted from the lower branching index values. Moreover, incorporation of poly (propylene glycol) mono butyl ether as the mono functional long chain end capper resulted in lowering the glass transition temperature of the low $T_{g}$ polyesters. Adhesive testing of the samples using peel testing. Size exclusion chromatogram of three different polymers is shown in Figure 1-3 with polydispersity index.

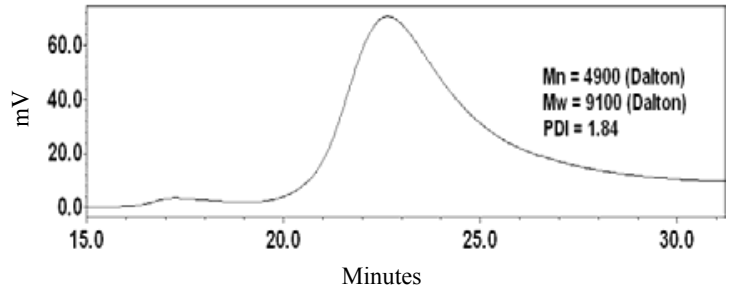

Figure 1. Poly(propane1,3-diol fumaric acid 1,4-cyclohexanedimethanol) (PPFCDM)

As the molecular weight increases mechanical strength increases. At low temperatures the polymer behaves like a glass. Molecular weights of these polymers are low and hence these cannot find applications as acrylic polymers where they are used as adhesives.

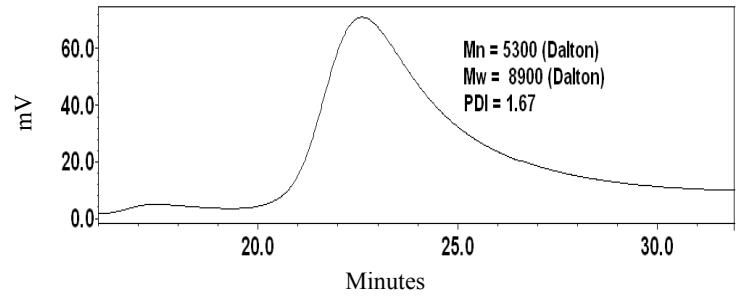

Figure 2. Poly(propane1,3-diol fumaric acid1,4-cyclohexanedicarboxylic acid) (PPFCDC)

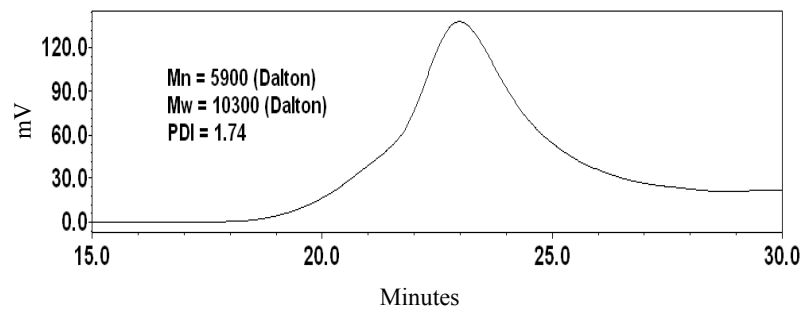

Figure 3. Poly(1,4-cyclohexanedimethanol fumaric acid 1,4-cyclohexanedicarboxylic acid) (PCDMFCDC) 


\section{Viscosity measurement}

The inherent viscosity of the resulting polymers was determined in dimethyl sulphoxide solution at $30{ }^{0} \mathrm{C}$ using Ubbelohde viscometer. In each case, $25 \mathrm{mg}$ of dry pure copolyester sample was dissolved in $25 \mathrm{~mL}$ of DMSO, kept aside for some time with occasional shaking. The $\eta_{\text {inh }}$ was calculated from the flow time measurement. The inherent viscosity values of all the copolyesters are listed in Table 2. The data reveals that these polymers are of reasonably high molecular weight. The viscosities of solutions of the polymers in chloroform were measured at $25{ }^{\circ} \mathrm{C}$ using an Ubbelohde viscometer. The intrinsic viscosity $(\mathrm{g})$ was calculated from these measurements. The complex dynamic viscosities $g$, of the copolyester melts were recorded using a Rheomotrics mechanical spectrometer RMS-605, operating in the dynamic shear mode between two parallel plates at $130{ }^{\circ} \mathrm{C}$. The frequency was varied from 0.1 to $100 \mathrm{rad} / \mathrm{s}$. The radius of the samples was $25 \mathrm{~mm}$ and the thickness was about $1.0 \mathrm{~mm}$. The samples were prepared by press molding at $130{ }^{\circ} \mathrm{C}$.

Table 2. Intrinsic viscosity data of random co-polyesters

\begin{tabular}{cccc}
\hline Polymers & PPFCDC & PPFCDM & PCDMFCDC \\
\hline Intrinsic viscosity, dL/g & 0.52 & 0.50 & 0.63 \\
\hline
\end{tabular}

One of the best known viscosity-molecular constitution relationship proposed by Dunstan is

$$
\log \eta=\mathrm{aM}+\mathrm{b}
$$

where $\eta$ is viscosity, $M$ is Molecular weight and a $\&$ b are constants. The constant a is for all homologous series and $\mathrm{b}$ is a constitutive factor characteristic of a particular series.

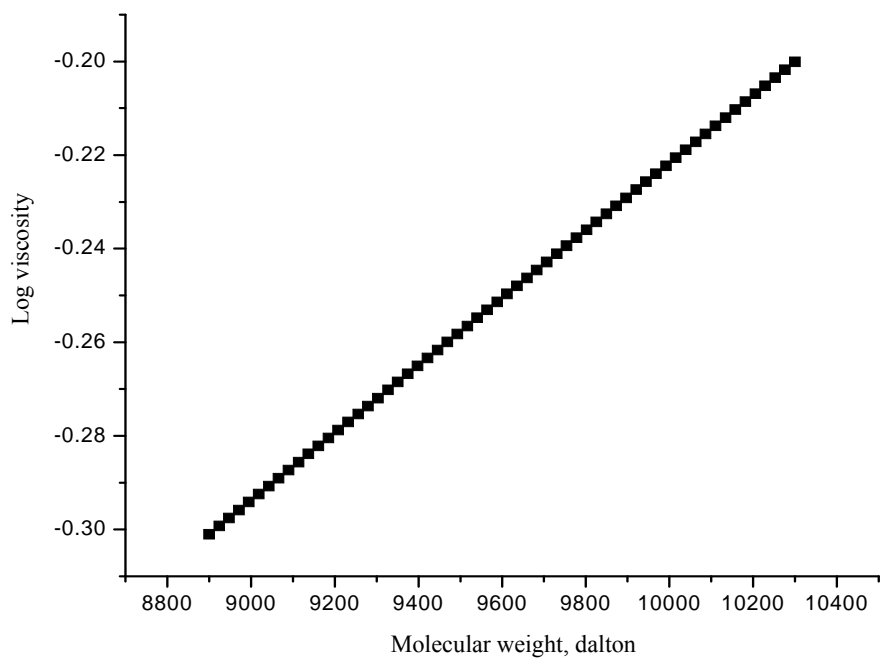

Figure 4. Shows the variation of viscosity versus molecular weight

From the graph (Figure 4) the value of ' $a$ ' is calculated as $7.4 \times 10^{-05}$ and the value of $b$ is found to be -0.32 . The constant value ' $a$ ' refers to homologous series of esters and the value of ' $b$ ' indicates the value of copolyesters of the particular compound pertaining to the present investigation.

Figure 5 and 6 show the variations of viscosity and molecular weight with three different copolyesters. General trend is increasing with three different copolyesters. 


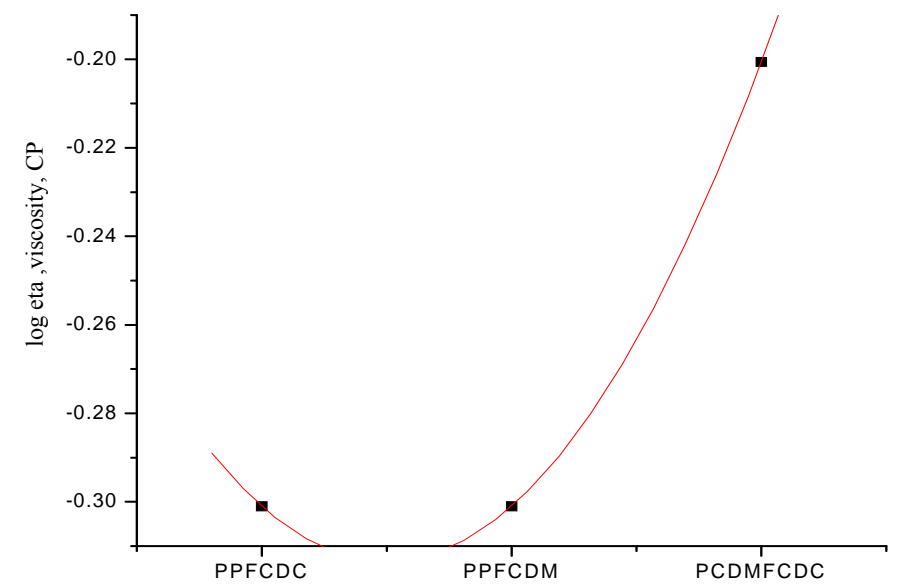

Figure 5. The variations of viscosity with three different copolyesters

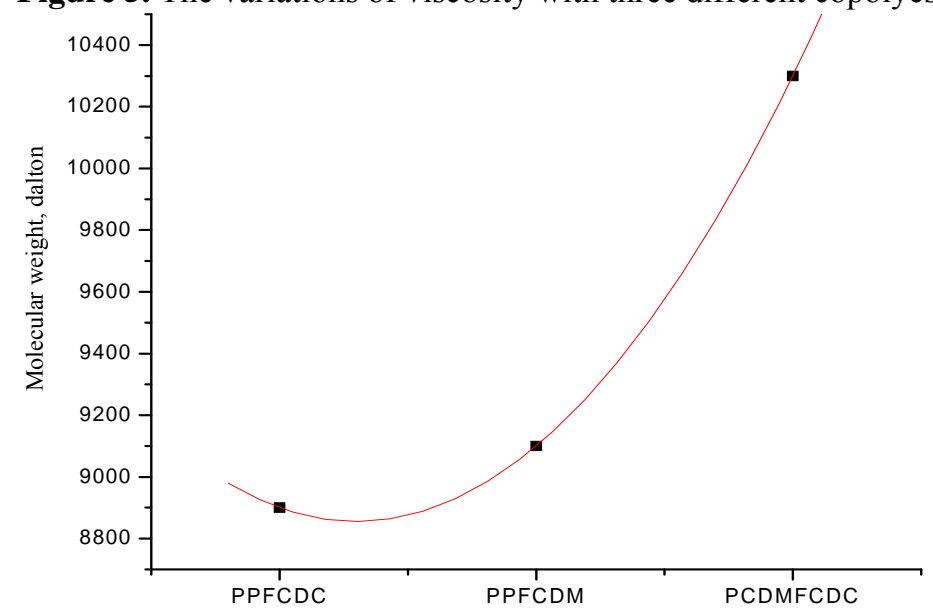

Figure 6. The variations of molecular weight with three different copolyesters

\section{Solubility test}

All the synthesised polymers were easily soluble in aprotic polar solvents like tetrahydrofuran, dimethyl sulphoxide, dimethyl formamide, dimethylacetamide, dioxane, and pcresol and in chlorinated solvents such as methylene dichloride, chloroform and insoluble in toluene, $n$-hexane, benzene, xylems, diethyl ether and other hydrocarbons solvents. This may be due to the inter-molecular interactions of polar solvents with ester linkage of the polymer molecules. Some of the polymers do not dissolve in the above said solvents; which polymer does not dissolve, in which other solvent it is dissolving. This might be due to the high rigid aromatic nature of the polymers ${ }^{10-11}$.

Solubility of copolymers was determined by dissolving $0.5 \mathrm{~g}$ of samples in $10 \mathrm{~mL}$ of chloroform or a trichloroacetic/chloroform mixed solvent at room temperature. The solubility is indicated by number of ' + '. Essentially, the samples were first dissolved in chloroform. If a complete dissolution is achieved within $10 \mathrm{~min}$ at ambient temperature, solubility is rated as ' +++ '. 
For samples taken longer time to completely dissolve in chloroform, the solubility was measured in a trichloroacetic acid/chloroform mixed solvent. The rating of ' ++ ' and '+' indicates that the samples are fully dissolved in $2 \%(\mathrm{w} / \mathrm{w})$ and $5 \%$ of trichloroacetic acid/chloroform mixed solvent within $10 \mathrm{~min}$, respectively.

Table 3. Solubility data of random copolyesters

\begin{tabular}{|c|c|c|c|c|c|c|c|c|c|c|c|}
\hline $\begin{array}{l}\dot{z} \\
\dot{r}\end{array}$ & Polymer & $\begin{array}{l}0 \\
\stackrel{0}{0} \\
\stackrel{0}{0} \\
\text { \& }\end{array}$ & 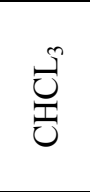 & 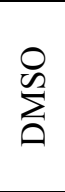 & $\underset{\Xi}{\stackrel{\Xi}{\Xi}}$ & $\begin{array}{l}\bar{O} \\
\text { 量 } \\
\sum\end{array}$ & 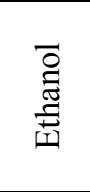 & 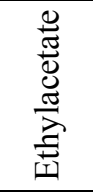 & 刍 & $\sum_{\dot{\theta}}^{L}$ & $\begin{array}{l}\overline{ \pm} \\
\overline{3} \\
3\end{array}$ \\
\hline 1 & PPFCDC & +++ & +++ & ++ & $-\quad+$ & - & +++ & +++ & ++ & $-\quad+$ & - \\
\hline 2 & PPFCDM & +++ & +++ & ++ & $-\quad+$ & -- & - - & ++ & ++ & ++ & -- \\
\hline 3 & PCDMFCDC & +++ & +++ & ++ & $-\quad+$ & -- & -- & ++ & ++ & ++ & -- \\
\hline
\end{tabular}

\section{Conclusion}

The MW-assisted synthesis of copolyesters from propane1, 3-diol and 1, 4-cyclohexane dimethanol with various carboxylic acids in the presence of titanium isopropoxide as the catalyst was performed. In order to improve the thermal and mechanical properties of the poly(estercarbonate)s, chain-extension products (PCDMFCDC, PPFCDM and PPFCDC) were also synthesized starting from dihydroxy-terminated oligomers obtained by the etherification of succinic acid in the presence of 1,3-propanediol and 1,4-cyclohexanedimethanol (PCDMFCDC, PPFCDM and PPFCDC). Characterization of the molecular weight and branching index indicated that synthesis of long chain branched low $\mathrm{T}_{\mathrm{g}}$ polyesters in the presence of mono functional reagents retarded gel formation and permitted synthesis of gel free polymers having higher degrees of branching, intrinsic viscosity data of random copolyesters, $0.52,0.50$ and 0.63 .

\section{References}

1. Carothers W H, J Am Chem Soc., 1929, 51(8), 2548-2559; DOI:10.1021/ja01383a041

2. Edlund U and Albertsson A C, Advanced Drug Delivery Review, 2003, 55(4), 585609; DOI:10.1016/S0169-409X(03)00036-X

3. Carothers W H and Arvin G A, J Am Chem Soc., 1929, 51(8), 2560-2570; DOI:10.1021/ja01383a042

4. Carothers W H and Van Natta F J, J Am Chem Soc., 1930, 52(1), 314-326; DOI:10.1021/ja01364a045

5. $\quad$ Manabe K, Sun X M and Kobayashi S, J Am Chem Soc., 2001, 123(41), 10101-10102; DOI:10.1021/ja016338q

6. Manabe K, Limura S, Sun X M and Kobayashi S, J Am Chem Soc., 2002, 124(40), 11971-11978; DOI:10.1021/ja026241j

7. Kobayashi S, Uyama H, Suda S and Namekawa S, Chem Lett., 1997, 26(1), 105; DOI:10.1246/cl.1997.105

8. Patil C B, Mahajan S K and Katti S A. J Pharm Sci Res., 2009, 3(1), 11-22.

9. Liu Y, Ranucci E, Lindblad M S and Albertsson A C, J Polym Sci Pol Chem., 2001, 39(14), 2508-2519; DOI:10.1002/pola.1227

10. Murugavel S C, Swaminathan C S and Kannan P, Polymer, 1997, 38(20), 5179-5183; DOI:10.1016/S0032-3861(97)00040-2

11. Murugavel S C, Kaliyappan T, Swaminathan C S and Kannan P, J App Poly Sci., 1997, 65(11), 2151-2157; DOI:10.1002/(SICI)1097-4628(19970912)65:11<2151::AIDAPP11>3.0.CO;2-6 